UCRL-JC-122313

PREPRINT

\title{
LONGITUDINAL DYNAMICS AND STABILITY IN BEAMS FOR HEAVY-ION FUSION
}

\author{
W. M. Sharp \\ D. A. Callahan \\ D. P. Grote
}

\section{MECENM \\ APR 18 MOPS \\ OSTI}

This paper was prepared for submittal to the 8th ICFA Advanced Beam Dynamics Workshop on Space Charge Dominated Beams and Applications of High Brightness Beams Bloomington, IN

October 10-13, 1995

January 5, 1996

This is a preprint of a paper intended for publication in a journal or proceedings. Since changes may be made before publication, this preprint is made available with the understanding that it will not be cited or reproduced without the permission of the author. 


\section{DISCLAIMER}

This document was prepared as an account of work sponsored by an agency of the United States Government. Neither the United States Government nor the University of California nor any of their employees, makes any warranty, express or implied, or assumes any legal liability or responsibility for the accuracy, completeness, or usefulness of any information, apparatus, product, or process disclosed, or represents that its use would not infringe privately owned rights. Reference herein to any specific commercial product, process, or service by trade name, trademark, manufacturer, or otherwise, does not necessarily constitute or imply its endorsement, recommendation, or favoring by the United States Government or the University of California. The views and opinions of authors expressed herein do not necessarily state or reflect those of the United States Government or the University of California, and shall not be used for advertising or product endorsement purposes. 


\title{
Longitudinal Dynamics and Stability in Beams for Heavy-Ion Fusion*
}

\author{
W. M. Sharp, D. A. Callahan, and D. P. Grote \\ Lawrence Livermore National Laboratory L-440, Livermore, CA 94550, USA
}

\begin{abstract}
Successful transport of induction-driven beams for heavy-ion fusion requires careful control of the longitudinal space charge. The usual control technique is the periodic application of timevarying longitudinal electric fields, called "ears," that, on the average, balance the space-charge field. This technique is illustrated using a fluid/envelope code CIRCE, and the sensitivity of the method to errors in these ear fields is illustrated. The possibility that periodic ear fields also excite the longitudinal instability is examined.
\end{abstract}

\section{Introduction}

Since its inception in the 1970s, the American heavy-ion fusion (HIF) program has favored induction accelerators. [1,2] These devices can accelerate much higher currents than the radio-frequency (rf) accelerators preferred in Europe, and they can compress the beam during acceleration, simplifying the problem of longitudinal compression. However, to be economically competitive, induction accelerators must carry nearly the maximum transportable charge along the entire lattice, so that beam dynamics in such devices is invariably dominated by space charge.

The effects of space charge on transverse dynamics are familiar and will not be discussed here in detail. Strong focusing is needed to balance the space-charge force, and this is usually applied by alternating-gradient (AG) electric or magnetic quadrupoles [3]. Since the space-charge force partially cancels the focusing force, space-charge-dominated beams are often substantially tune depressed [4], and since they maintain a nearly uniform charge density except near the edges, such beams have radially density profiles that are much flatter than the Gaussian profiles typically found in rf accelerators [5]. The space-charge fields for these flatter density profile are more nearly linear in the beam interior than an emittance-dominated beams, reducing the rate that transverse fluctuations thermalize. Finally, the transverse space-charge provides a mechanism for halo formation when a beam is imperfectly matched [6].

There are also two important longitudinal effects of space charge. Unlike radiofrequency accelerators, the accelerating fields of induction accelerators provide no longitudinal focusing, so space charge causes the beam to lengthen in the absence of supplemental focusing. Longitudinal control is typically maintained by time-varying electric fields, referred to here as "ears," that are added to the acceleration field in at least some induction cells to balance the space-charge force. The other longitudinal effect of space charge is an interaction, known as the "longitudinal instability," between the beam and its resistively retarded image field. This paper will discuss theoretical work on the generation and application of ear fields, and it will review simulations examining whether the periodic application of ear fields is likely to stimulate the longitudinal instability.

\footnotetext{
* The research was performed under the auspices of the U. S. Department of Energy by Lawrence Livermore
} National Laboratory under Contract No. W-7405-ENG-48. 


\section{Effects of Ear Fields}

\section{A. Time Dependence of Ears}

For the ion beams considered for heavy-ion fusion (HIF), which are typically meters long and only a few centimeters in radius, the longitudinal space-charge field is a highly non-linear function of position in the beam frame, so the ear fields must vary in time proportionally with the space-charge field as the beam moves through the acceleration gaps. The ideal ear field is related to the longitudinal component $E_{z}$ of the beam spacecharge field at corresponding times by

$$
E_{e a r} \approx-\eta_{\text {ear }}\left\langle E_{z}\right\rangle
$$

where the ear occupancy $\eta_{e a r}$ is the ratio of the induction-cell gap length to the distance between those cells in which ears are applied, termed "ear cells." The angle brackets on $\left\langle E_{z}\right\rangle$ denote a density-weighted average over the beam cross-section. This average is needed because the impulse an ion receives from an induction cell is approximately independent of the transverse location of the particle, making it impossible for the ear field to nullify the space charge at all points across the beam. Using ears calculated from the averaged space-charge field minimizes this inevitable error in longitudinal confinement.

The average axial space-charge field $\left\langle E_{z}\right\rangle$ in Eq. (1) is found to depend significantly on both the current and radius profiles of the beam. This dependence is illustrated by fields calculated for a quadratically decreasing current at the beam ends in Fig. 1 and for a Gaussian current fall off in Fig. 2. In each case, the beam is presumed to be centered in a perfectly conducting cylindrical pipe, and the parameters are those of a small recirculating induction accelerator [7] being developed at the Lawrence Livermore National Laboratory (LLNL), except that the beam is assumed here to the axisymmetric and the beam midsection has been shortened to highlight field changes near the ends. The maximum current $I_{b 0}$ is $2 \mathrm{~mA}$ of singly charged potassium ions with an energy of $80 \mathrm{keV}$, and the maximum radius $a_{0}$ of the beam is $0.011 \mathrm{~m}$. For each current profile, radius profiles are shown for several choices of the emittance variation, corresponding to a uniform beam radius $a$, a uniform transverse emittance $\epsilon_{\perp}$, a uniform transverse temperature $\epsilon_{\perp} / a$, and a uniform charge density $I_{b} /\left(\pi \beta c a^{2}\right)$, where $\beta$ is the beam axial velocity scaled by the speed of light $c$. Although these simple profiles are unlikely to match that in an experimental beam, they illustrate the sensitivity of the space-charge field to the beam radial variation. Figs. 1a and $2 a$ show the relative radial profiles of the beams for these various emittance choices, and the corresponding current profiles are plotted as dashed lines.

In the field plot for the "quadratic-end" cases in Fig. 1b, one finds that the peak space-charge field increases for profiles that have smaller end radii, with the field for a uniform-density beam being more than twice that for a uniform-radius beam. It is also evident that $\left\langle E_{z}\right\rangle$ for the uniform-density is qualitatively different from the others. For the cases with a finite beam-end radius, the field magnitude is seen to drop significantly in a narrow region at the beam end. This region has a characteristic length of $0.415 R$ for a pipe with radius $R$, and within the region, the absence of charge beyond the beam end reduces the axial field. At the endpoints, the field is reduced by approximately half. In contrast, $\left\langle E_{z}\right\rangle$ for the uniform-density case varies monotonically near the end due to the rapidly decreasing radius. The field plots for the "Gaussian-end" cases in Fig. are substantially different. As expected from the analytic expressions for $\left\langle E_{z}\right\rangle$ in Ref. [8], the 

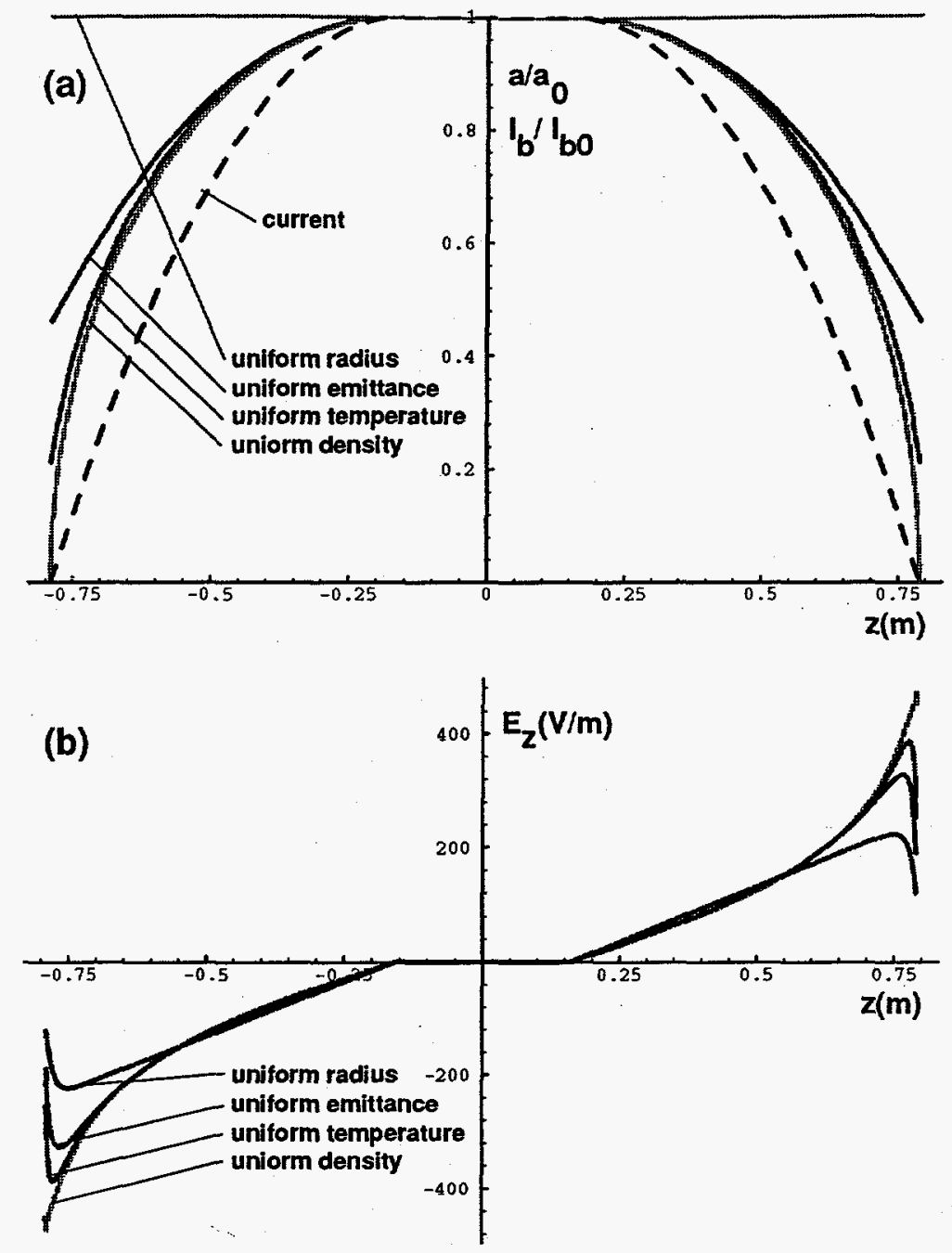

Fig. 1 (a) Radial profiles and (b) radially averaged space-charge fields for axisymmetric beams with quadratically decreasing current $I_{b}$ near the ends and various choices of emittance variation. Current profile is plotted as a dashed line.

peak field magnitudes occur near the points of maximum slope in the beam-current, with some shift in magnitude and position arising from the radius variation. However, since there is less difference in the beam radii at these points than in the quadratic-end cases, the peak space-charge field differs by only about $5 \%$ between the various emittance profiles. Also, the rapid fall off in field strength near the ends is not seen in these cases. These two cases show that a detailed knowledge of the beam current and radius profiles is important in determining both the waveform and range of variation needed for ear generation.

Despite the assumption of axisymmetry used in calculating the fields in Figs. $1 \mathrm{~b}$ and $2 \mathrm{~b}$, the results are nearly the same both for beams displaced from the accelerator axis and for beams in AG lattices. Using a three-dimensional simulation code WARP3d [9], we find for $a / R=0.33$ that values of $\left\langle E_{z}\right\rangle$ for a centered beam and one displaced $0.14 R$ from the axis differ by no more than $2 \%$. Similarly, the average space-charge field of a beam with a circular cross-section has been compared with that of an elliptical beam with major and minor radii $a$ and $b$ by matching the circular-beam radius to the geometric mean $(a b)^{1 / 2}$. For $a / b=1.8,\left\langle E_{z}\right\rangle$ differs from the field for the corresponding round beam by at most $4 \%$. 

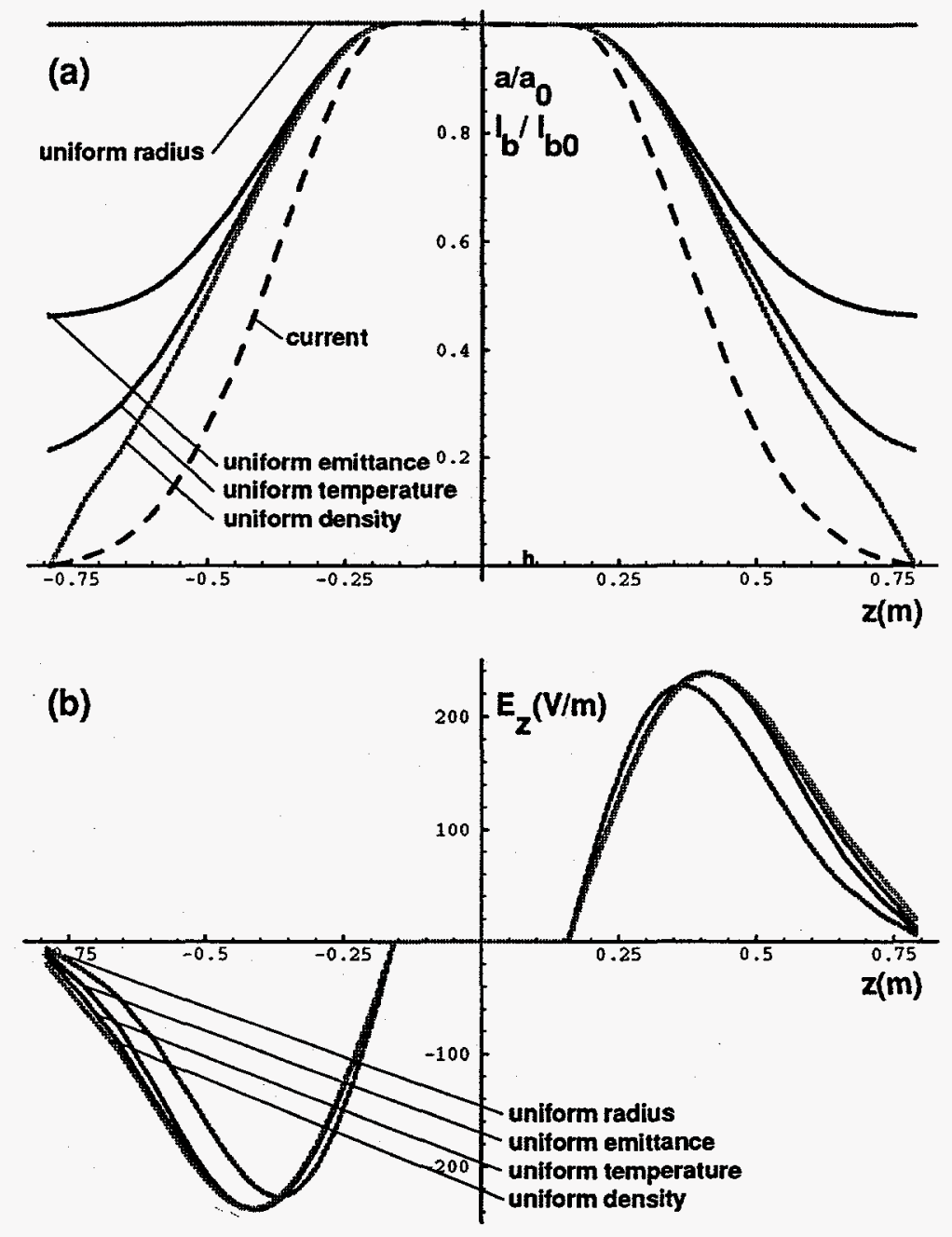

Fig. 2 (a) Radial profiles and (b) radially averaged space-charge fields for axisymmetric beams with Guassian decrease in current near the ends and various choices of emittance variation. Current profile is plotted as a dashed line.

Furthermore, since $a$ and $b$ fluctuate in the AG focusing on a period that is typically much smaller than the characteristic longitudinal-expansion time, the effect of beam ellipticity on longitudinal dynamics is substantially less than expected from this static comparison.

\section{B. Calculation of Optimal Ears}

The fluid/envelope code CIRCE [10] is used here to assess the effects of space charge on the longitudinal dynamics of heavy-ion beams. Longitudinal dynamics is modeled in the code by treating slices of the beam as Lagrangian fluid elements. The boundaries between slices are assumed to be perpendicular to the beam axis and to respond to the average space-charge field $\left\langle E_{z}\right\rangle$ and to the axial field from acceleration cells. The neglect in this model of radial variation in the space-charge field is justified because this variation remains small so long as the space-charge potential across a beam is large compared with the transverse thermal energy, a condition that characterizes space-charge-dominated beams. The beam can be initialized using any of the emittance profiles discussed in section IIA, and the average space-charge field can optionally be calculated from the analytic expressions 
in $\operatorname{Ref}[8]$.

Optimal ears are generated by a simple procedure. For a particular beam and lattice, CIRCE is run once without ear fields and with the axial space-charge force artificially switched off. This condition is equivalent to having perfect ears that are applied continuously. During the run, the average axial space-charge field $\left\langle E_{z}\right\rangle$ is calculated as the beam enters each ear cell, and Eq. (1) is used to obtain the corresponding ear fields, which are written along with timing data to an external file. For beams with a finite emittance and radius at the ends, $\left\langle E_{z}\right\rangle$ is estimated by the simple expression

$$
\left\langle E_{z}(z)\right\rangle \approx-\frac{1}{4 \pi \epsilon_{0}}\left\{\left[\frac{1}{2}+\ln \left(\frac{R^{2}}{a b}\right)\right] \frac{\partial \lambda}{\partial z}-\frac{\lambda}{2 a b} \frac{\partial(a b)}{\partial z}\right\}
$$

where $\lambda=I_{b} /(\beta c)$ is the beam line-charge density, $R$ is the beam-pipe radius, and $z$ is axial position in the beam rest frame. A more complicated Bessel-series form from Ref. [8] is used for beams with uniform charge density. Even though Eq. (2) misses the rapid field variation near the ends seen in Fig. $1 \mathrm{~b}$, it is used here because the high frequency response needed to balance the space-charge field near the ends is beyond current pulse-generation technology. On subsequent runs, ears are obtained from the external file, and the spacecharge field, including any rapid variation at the ends, is calculated at every step from the appropriate expression from Ref. [8].

\section{Dynamics with Optimal Ears}

The effects of optimal ears are illustrated here using the lattice and initial beam parameters of the small recirculating induction accelerator being developed at LLNL [7]. As in the field calculations of section IIA above, the initial current of singly charged potassium ions is $2 \mathrm{~mA}$, but the emittance is sufficiently low that at the initial energy of 80 $\mathrm{keV}$, the beam transverse dynamics is space-charge dominated. In the uniform-emittance cases presented here, the beam doubles its energy in 15 laps while compressing by a factor of four in duration and a factor of two in length. A head-to-tail velocity variation or "tilt" of about $35 \%$ is imposed during the first lap by triangular accelerating pulses in the first ten active cells, and ear fields are applied in each $36 \mathrm{~cm}$ half-lattice period (HLP), except in two sections of three HLPs each that are used for insertion and extraction of the beam. The initial pulse duration is $4 \mu \mathrm{s}$, and the current decreases quadratically to zero in $1 \mu \mathrm{s}$ at both ends.

In the absence of ears, space charge is seen in Fig. 3a to push out the ends, and rarefaction waves move in from each end at the electrostatic wave speed

$$
C_{s} \approx\left(\frac{g q e \lambda}{4 \pi \epsilon_{0} M}\right)^{1 / 2} \ll \beta c,
$$

where $q$ is the ion charge state, $M$ is the ion mass, and $g=\ln \left(R^{2} / a^{2}\right)$ is a factor accounting for the wall geometry. The velocity tilt imposed by the first ten cells causes the density at the beam center to increase until, in the fourth lap, the rarefaction waves meet, and after that point the entire beam elongates to about 2.5 times the initial value. This case indicates that a beam of length $L_{b}$ can be transported without ear fields only over lattice lengths that are short compared with $\beta c L_{b} /\left(2 C_{s}\right)$. 

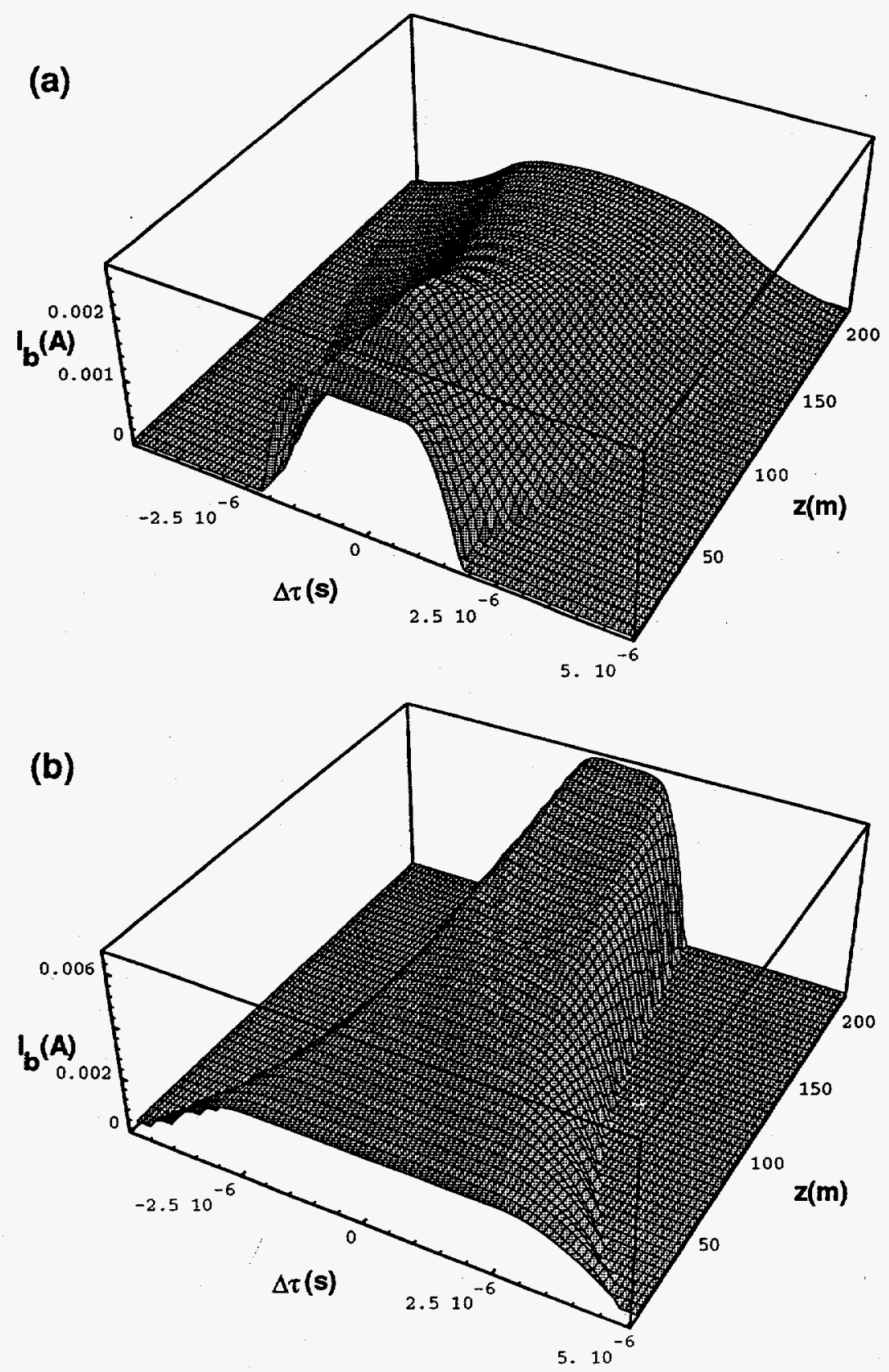

Fig. 3 CIRCE calculation of beam-current histories of a space-charge-dominated beam (a) without "ear" fields and (b) with space charge balanced by optimal ears applied periodically. Current is plotted as a function lattice position $z$ and time $\Delta \tau$ relative to the beam midpoint. Ripples seen in (b) near $z=0$ are a plotting artifact.

With the axial component of the space-charge field set to zero, the beam current is found to compress in a nearly self-similar manner. Using ears generated by this first run, a second case, including space charge, gives the current history shown in Fig. $3 \mathrm{~b}$. On the scale shown, the pulse history is indistinguishable from that for the run without a longitudinal space-charge field, although more detailed plots show some low-amplitude space-charge waves launched during the first few laps. These waves result from the initial mismatch near the ends between the monotonically varying ear field and the rapidly varying 
space-charge field seen in Fig. 1b for a uniform emittance beam. Examination of the beam line-charge indicates, however, that the ends of the beam are quickly compressed, making the space-charge field approximately match the average ear field and effectively ending the generation of space-charge waves. A similar adaptation of the beam to the ear field is found with other beam emittance profiles.

If the optimal ears for a compressing beam are applied but no velocity tilt is imposed by the accelerating voltage, then the beam ends are pushed in according to the compression schedule, but large space-charge waves are generated as the ear fields move into the denser part of the beam. After six laps, the waves collide in a highly non-linear fashion that is not modeled correctly by CIRCE, illustrating that a velocity tilt is necessary for quiescent beam compression, as well as suitable ear fields.

Of necessity, the ear fields are applied periodically, so the ends are repeatedly being pushed in and then expanding. However, provided that this period is short compared with the characteristic time for beam expansion, these kicks are not an important source of noise. This consideration imposes a constraint on the maximum spacing of ear cells, but in practice, the breakdown voltage in induction cells is typically a more stringent limitation.

\section{Dynamics with Imperfect Ears}

Due to the imprecision in measuring the beam current profile and limitations in waveform generation, the ear fields used in an experiment are certain to be imperfect. A number of runs have been made to test qualitatively the sensitivity of beam dynamics to ear-field errors. For cases in which ear fields generated from one beam profile are applied to a beam with a different profile, the beam is found to be rather insensitive to the discrepancy for any pairing that involves the uniform emittance, density, or transverse temperature profiles discussed in section IIA. The reason for the insensitivity is that mismatches between the average ear fields in these cases and the space-charge field become appreciable only in the low-density region at the ends, where $\lambda$ is less than about $10 \%$ of its mid-pulse value. In such cases, that beam is found to adapt to discrepancies as large as $60 \%$ between the ear and space-charge fields. Appreciable space-charge waves are generated, however, in beams with a uniform initial radius controlled by ears from one of the other profiles. As is evident from Fig. 1b, the space charge for a uniform-radius beam differs visibly from that for the other profiles over almost half of the current rise and fall lengths, and the size of the field discrepancy is larger than in the previously cited cases. Both differences are expected to increase wave generation, but the effects have not been isolated and quantified.

A second series of runs was made using current profiles that differ from that used to generate the ear fields. Fig. 4a shows the current history of a beam with $1.2 \mu$ s current rise and fall times confined by ears calculated for a $1 \mu$ s rise and fall. In this case, the mismatch between the average ear field and the space-charge field occurs near the current flat-top, and sizable linear space-charge waves are generated. The waves are seen to increase in amplitude with $z$ because the fluid model used in CIRCE for longitudinal dynamics has no damping, so the wave energy is trapped in a progressively shorter length as the beam compresses. In an experiment or particle simulation, some damping is expected due to phase mixing and thermalization, and there would be a corresponding growth in longitudinal temperature. A more extreme mismatch is generated by specifying a cubic fall-off in the beam current near the ears but using ears calculated for a quadratic drop-off. The result, shown in Fig. 4b, is that larger linear waves are initially generated and become 

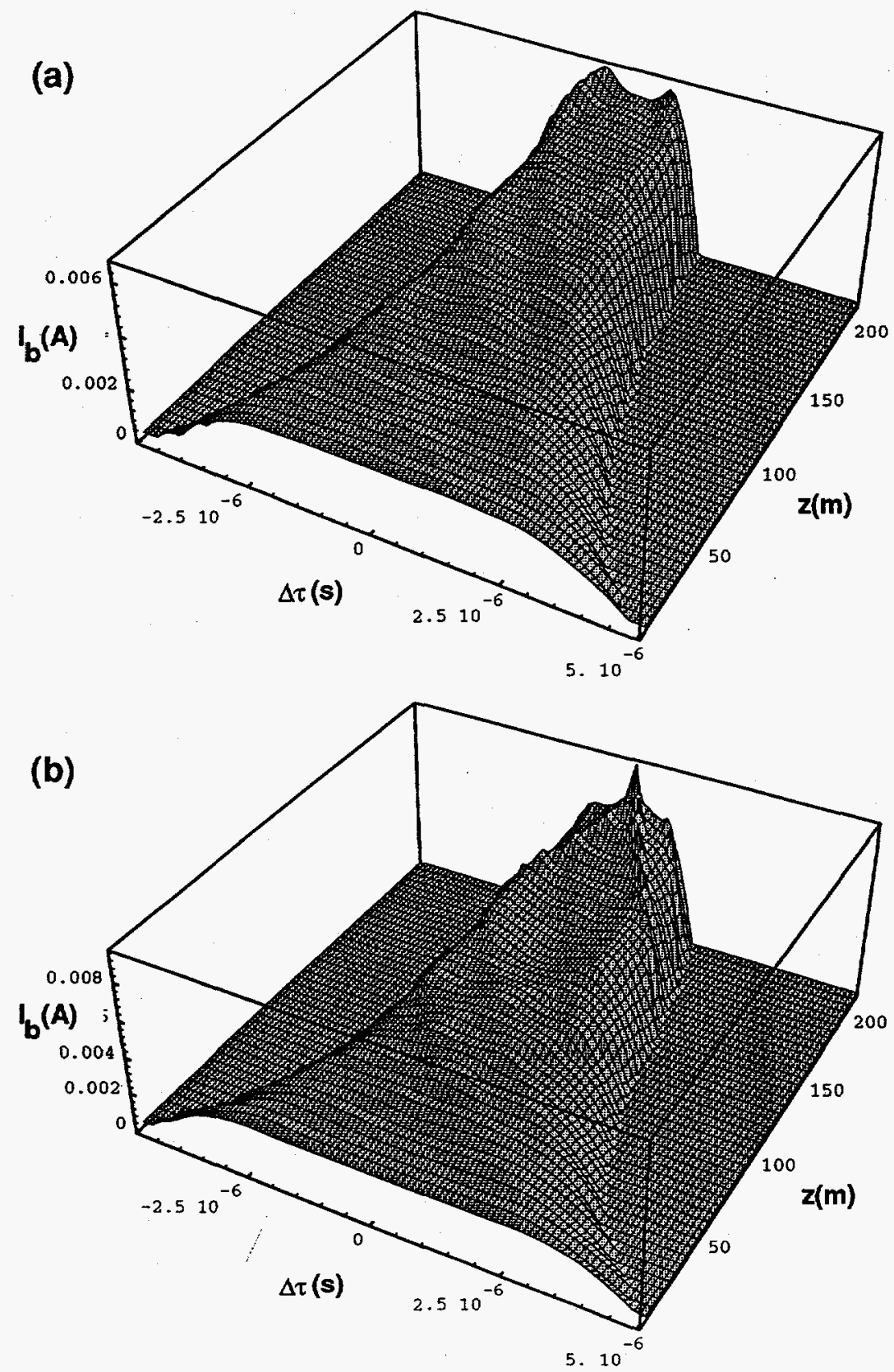

Fig. 4 CIRCE calculation of space-charge waves generated (a) by inappropriate current rise and fall times and (b) by an inappropriate current-profile form

nonlinear after 15 laps. Both cases in Fig. 4 indicate that the beam is moderately sensitive to errors in the ear field that occur in the higher-density regions of the beam.

\section{Longitudinal Instability}

\section{A. Mechanism}

The longitudinal instability is a concern in HIF drivers because it amplifies small perturbations launched from the beam head. These perturbations may be caused by errors 
in the acceleration or ear fields, or at a low level, by the periodic application of ear fields. The instability has the same mechanism exploited in "resistive-wall" amplifiers, except that the impedance in HIF accelerators results mainly from the accelerating modules. The instability can be stabilized by a sufficiently large spread in longitudinal momentum, but the required spread exceeds the limit imposed by chromatic aberration in the focusing lens system.

The longitudinal instability can be modeled using a simple fluid model. If we consider an incompressible axisymmetric beam with radius $a$ traveling down a pipe of radius $R$, one-dimensional (1-D) linear cold-fluid theory shows that two waves will develop: a "fast" wave traveling forward in the beam frame and a "slow" wave traveling backward. These waves propagate with a phase velocity in the beam frame near the electrostatic wave speed $C_{s}$, given by $\mathrm{Eq}$. (3). In induction drivers, the resistance of acceleration modules causes the beam image charge to lag behind the beam, and the interaction of perturbations with their retarded images leads to the growth of slow waves, as well as damping of fast waves. In early theoretical work on the instability [10], module impedance was modeled as a distributed resistance per unit length $\eta$, and the 1-D cold-fluid model predicted a dangerously high growth rate $\Gamma$ and a growth length

$$
\frac{C_{s}}{\Gamma} \approx \frac{g}{2 \pi \epsilon_{0} \eta \beta c}
$$

that was much shorter than the beam length. A more sophisticated 1-D model [13], which includes a realistic distributed capacitance in parallel with the resistance, shows a much lower maximum growth rate, occurring for a perturbation wavelength that is large compared with the pipe radius $R$.

\section{B. Simulations}

An $r-z$ branch of the WARP code was developed to model the longitudinal dynamics of HIF drivers [14]. This code is a $21 / 2$-dimensional, cylindrically symmetric particlein-cell code. Field calculation is done in a window that moves with the beam center of mass. In this computational window, the fields are treated as purely electrostatic, since magnetic fields arise only from longitudinal temperature and any velocity tilt, and they affect dynamics only like the square of these relative velocities scaled by $c^{2}$. To allow study of the longitudinal instability, a wall model with distributed resistance and capacitance in parallel is used. This approximation for the induction modules contains the relevant physics, and also corresponds well with recent analytic work [13]. The resistivewall contribution to the electric field is calculated using a Poisson solve at the boundary because this approach is smoother and more physical than using the explicit beam current.

Simulations with a purely resistive wall in which a perturbation is launched from the beam head have shown growth of the backward traveling wave, as expected. The measured growth rate is lower than the prediction of cold-beam fluid theory by about $15 \%$. This discrepancy is probably due to the effects of finite transverse temperature, but the matter requires further research. The perturbation reflects off the beam tail, and some steepening of the perturbation is seen during reflection. This steepening appears to be a nonlinear effect since it is more evident in larger perturbations than in small ones. This nonlinear pulse narrowing is also accompanied by dispersion, so that the wave lengthens and decreases in amplitude due to the velocity spread between different frequency components as the perturbation travels from the beam tail to the head. 
When a capacitive component is included on the impedance, WARPrz simulations show both the lower growth rates and the longer wavelength of the fastest growing mode predicted by cold-beam fluid theory. For wavelengths that are comparable with the beam length perturbations are excited, but little growth is seen. These perturbations move back and forth from beam head to tail with little change in amplitude.

For a realistic appraisal of the effects of the longitudinal instability, finite-amplitude sources of perturbations need to be considered. One source of such perturbations is the intermittent application of the ear fields. In most of WARPrz simulations, ear fields are applied at each time step and are designed to keep the beam from expanding or contracting. In an experiment, these fields will be applied at fixed locations along the accelerator and the beam will contract and then expand between ear cells. CIRCE simulations with driver parameters show that a train of low-level perturbations is launched from the beam ends, and with a non-zero impedance, these perturbations will be amplified by the longitudinalinstability mechanism.

In the simulations reviewed here, each application of the ears consists of the following steps: (1) The beam is allowed to expand for $0.48 \mu \mathrm{s}(48 \mathrm{~m}$ at $0.33 \mathrm{c})$. (2) The expansion velocity is reversed by applying ear fields at the ends for $0.0875 \mu \mathrm{s}$. (3) The beam is allowed to expand for $0.48 \mu \mathrm{s}$. After these operations, the beam should be back to its original length.

In the absence of errors, the intermittent application of ears is found to be benign. Perturbations on the beam are minimized when an electric ear field is applied at each end that is proportional to the average particle velocity in the beam frame as a function of $z$ after the first expansion. The proportionality constant is varied until the beam is close to its original state just before the next application, and since the beam is being held at constant length, the same ear fields are used for each application. The simulation shows that these ear fields can be applied more than 20 times without significant perturbations developing on the beam, even in the presence of a $100 \mathrm{ohms} /$ meter resistive wall with no capacitance.[15]

Systematic ear-field errors are also found not to excite the instability. In a typical case, a error field with the form of half the period of a sine wave and a magnitude equaling $5 \%$ of the correct ear field was added to the. The same error was added at each application, making the ear fields systematically too large. Although such non-canceling ear errors were expected to be pathological, the beam adjusted quickly to the mismatched ears. The simulation was run for $1.4 \mathrm{~km}$, with 25 applications of the intermittent ears, but perturbations were excited only during the first few applications. The beam remained in its new equilibrium for the remainder of the simulation. Similar adjustment of the beam to mismatched ear fields has also been seen in longitudinal-control experiments done on the Single-Beam Test Experiment (SBTE) at LBNL[16]. In these experiments, ear fields of the form $E_{\text {ear }} \sim\left[1-\exp \left(-\alpha\left(t-t_{0}\right)\right]\right.$ were applied near the bearn tail, where $t_{0}$ is the time at which the beam current began to decrease in an ear cell, and $\alpha$ is an adjustable constant. No effort was made to match this ear waveform to the beam profile. The initial mismatch caused waves to be launched from the beam tail in the first few acceleration modules, but the beam soon reached a new steady-state configuration.

In contrast to the cases with systematic errors, random error are found to excite perturbations at each ear cell, with no adaptation of the beam profile. As an illustration, 


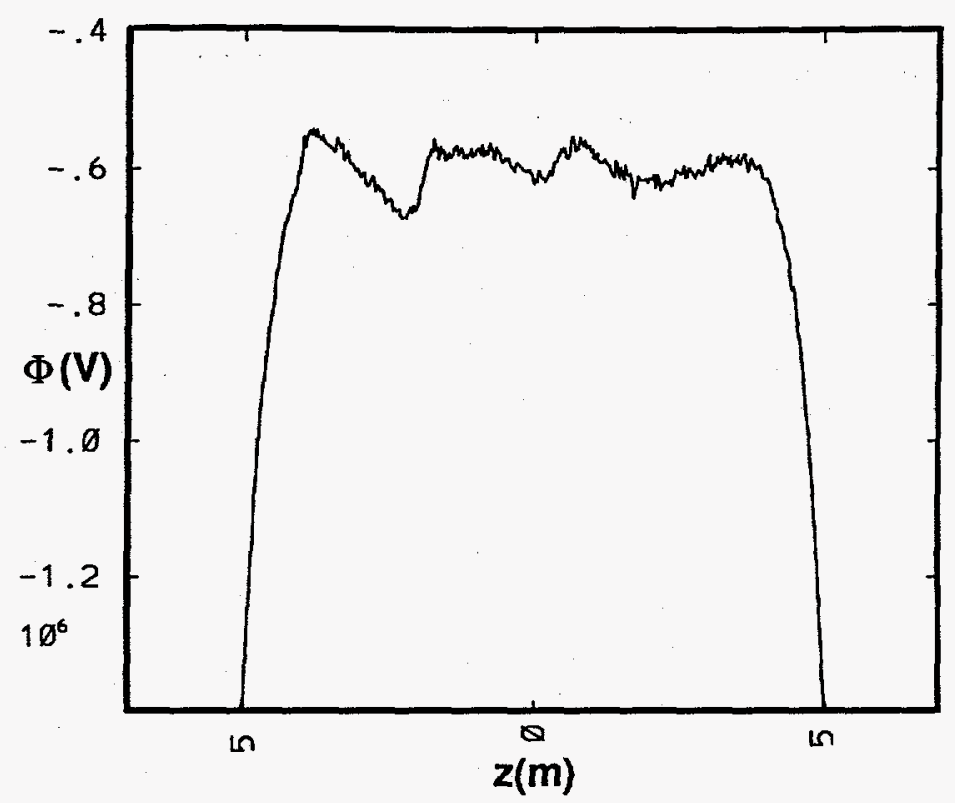

Fig. 4 WARPrz calculation of on-axis electrostatic potential of a driver-like beam after $1.4 \mathrm{~km}$ transport with random ear-field errors. Perturbations at the most unstable wavelength are excited.

ear errors were applied that had the same half-sine form used previously, but the error magnitude varied randomly between being $5 \%$ too large to $5 \%$ too small. In this case, a train of perturbations was launched from the beam head throughout the $1.4 \mathrm{~km}$ run, growing as expected as they convected toward the tail. The perturbation widths were found to approximately match the wavelength of the fastest-growing mode predicted by the cold-fluid analysis. Figure 4 shows the electrostatic potential on axis vs $z$ after 15 applications of the ear fields with $100 \mathrm{ohms} /$ meter wall resistance. Although the waves seen in Fig. 4 are sizable, it should be remembered that capacitance was deliberately omitted in this case to exaggerate the instability. A much smaller wave amplitude is found in more realistic cases.

\section{Conclusions}

The CIRCE simulations shown here illustrate that beams for heavy-ion fusion are moderately sensitive to errors in ear fields. Mismatches up to $50 \%$ between the average ear field and the beam space-charge field appears to be acceptable at the beam ends due to the low beam density there. However, near the high-density part of the beam, mismatches should not exceed about $20 \%$. The CIRCE results probably overestimate the wave amplitude due to mismatches due to the absence of phase-mix damping, but preliminary comparisons with the $3-\mathrm{D}$ particle simulation WARP3d indicate fair agreement nonetheless.

Simulations with an $r-z$ version of WARP indicate that the longitudinal instability is not a major problem for heavy-ion beams. The inclusion of a realistic induction-module capacitance gives a much lower growth rate than originally predicted from purely resistive models, and the intermittently applied ears do not normally seed the instability. Even the worst case, with random $5 \%$ errors in the absence of module capacitance, fails to excite 
destructive waves after $1.5 \mathrm{~km}$ of propagation. The modest growth of the longitudinal instability seen in realistic simulations can probably be stabilized by feed-forward correction, in which perturbations are detected at selected points along the accelerator, and correcting fields are applied downstream of the detectors.

\section{References}

[1] J. Hovingh, V. O. Brady, A. Faltens, D. Keefe, and E. P. Lee, Fusion Technol. '3, 255 (1988).

[2] R. O. Bangerter, Nuovo Cimento 106A,1445 (1993).

[3] M. Reiser, Theory and Design of Charged Particle Beams (John Wiley \& Sons, New. York, 1994), pp 162-165.

[4] E. P. Lee, T. J. Fessenden, and L. J. Laslett, IEEE Trans. Nucl. Science NS-32, 2489 (1985).

[5] M. Reiser and N. Brown, Phys. Rev. Lett. 71, 2911 (1993).

[6] R. L. Gluckstern, Phys. Rev. Lett. 73, 1247 (1994).

[7] A. Friedman, J. J. Barnard, M. D. Cable, D. A. Callahan, F. J. Deadrick, S. Eylon, T. J. Fessenden, D. P. Grote, H. A. Hopkins, V. P. Karpenko, D. L. Judd, H. C. Kirbie, D. B. Longinotti, S. M. Lunid, L. A. Nattrass, M. B. Nelson, M. A. Newton, T. C. Sangster, and W. M. Sharp, "Recirculating Induction Accelerators for Inertial Fusion: Prospects and Status," to be published in Fusion Eng. and Design.

[8] W. M. Sharp, A. Friedman, and D. P. Grote, "Effects of Longitudinal Space Charge in Beams for Heavy-Ion Fusion," to be published in Fusion Eng. and Design.

[9] A. Friedman, D. P. Grote, and I. Haber, Phys. Fluids B 4, 2203 (1992).

[10] W. M. Sharp, J. J. Barnard, D. P. Grote, S. M. Lund, and S. S. Yu, "Envelope Model of Beam Transport in ILSE" in AIP Conference Proceeding 297 (AIP Press, Woodbury, NY,1994), p. 540.

[11] C. K. Birdsall, G. R. Brewer, and A. V. Haeff, Proc. of the I. R. E. 41, ??? (1953).

[12] E. P. Lee, Proc. 1981 Linear Accel. Conf., Los Alamos Repost LA-9234-C, p. 263.

[13] E. P. Lee, Nuovo Cimentó 106A, 1679 (1993).

[14] D. A. Callahan, "Simulations of Longitudinal Beam Dynamics of Space-Charge Dominated Beams for Heavy Ion Fusion," PhD. Thesis, Lawrence Livermore National Laboratory Report UCRL-LR-119364 (1994).

[15] D. A. Callahan, A. B. Langdon, A. Friedman, and I. Haber, "Longitudinal Beam Dynamics for Heavy Ion Fusion Using WARPrz" in AIP Conference Proceeding 297 (AIP Press, Woodbury, NY,1994), p. 221.

[16] A. Faltens, LBL Half-Year Report LBL-19501, June 1985. 


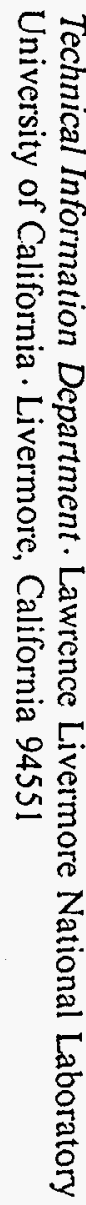

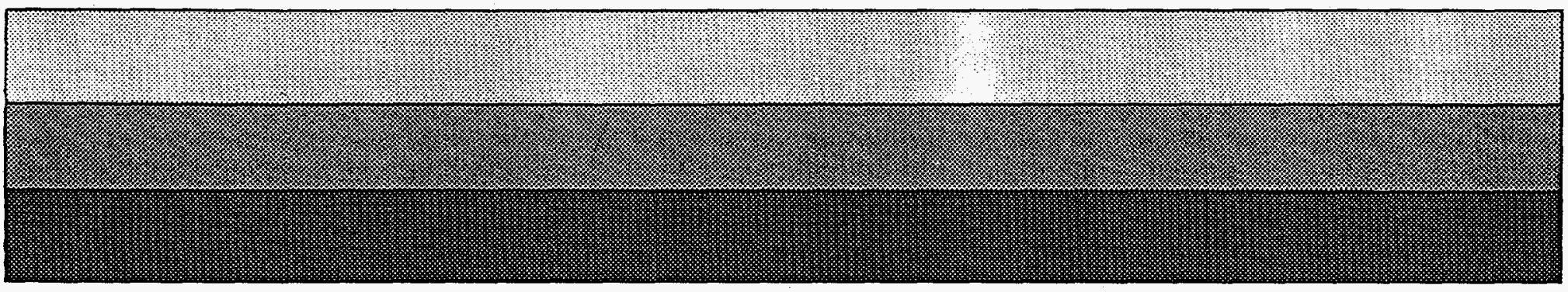

